\title{
Diacronie
}

Studi di Storia Contemporanea

$\mathrm{N}^{\circ} 15,3 \mid 2013$

Spazi, percorsi e memorie

\section{Enrica Salvatori (a cura di), Storia e territorio della} Val di Vara

Jacopo Bassi

\section{Q OpenEdition \\ Journals}

\section{Edizione digitale}

URL: http://journals.openedition.org/diacronie/645

DOI: 10.4000/diacronie.645

ISSN: 2038-0925

\section{Editore}

Association culturelle Diacronie

\section{Notizia bibliografica digitale}

Jacopo Bassi, «Enrica Salvatori (a cura di), Storia e territorio della Val di Vara », Diacronie [Online], № 15,

3 | 2013, documento 20, Messo online il 01 octobre 2013, consultato il 23 septembre 2020. URL :

http://journals.openedition.org/diacronie/645 ; DOI : https://doi.org/10.4000/diacronie.645 


\section{Diacronie}

N. 15 | 3|2013 Spazi, percorsi e memorie

20/

\section{RECENSIONE:}

\section{Enrica SALVATORI (a cura di), Storia e territorio della Val di Vara, Pisa, Felici, 2012,}

\section{4 pp.}

a cura di Jacopo BASSI *

Il volume - suddiviso in due tomi Storia e territorio della Val di Vara e I sentieri della Val di Vara - è parte di un più ampio progetto, sviluppato parallelamente in forma cartacea e online ${ }^{1}$, che ha coinvolto enti e professionalità diverse, espressione di ambiti disciplinari e amministrativi differenti, ma legati dal comune interesse per la valorizzazione - termine nato, riprendendo il concetto di mise en valeur francese, nel corso dei lavori della Commissione Franceschini del $1964^{2}-$ del territorio. Sino ad ora l'interesse per il complesso storico e paesaggistico della Val di Vara, territorio di confine sin dall'epoca bizantina, ha portato alla pubblicazione di prodotti editoriali "classici"3, spesso risultato di convegni ${ }^{4}$ o mostre 5 dedicate a questa porzione di territorio.

Il progetto, pertanto, è nato dal desiderio di creare uno strumento che potesse fungere al contempo da guida e apparato scientifico sul territorio della Val di Vara.

\footnotetext{
${ }^{1}$ URL: < http://www.tramontivaldivara.it/ > [consultato il 5 giugno 2013].

2 La Commissione Franceschini, istituita nel 1964, coniò il concetto di Beni culturali in Italia coniugandolo con quello di "storicità" degli stessi; il bene culturale, secondo quanto stabilito dalla legge, deve essere valorizzato. Sull'interpretazione del termine e sul dibattito scaturito si veda: CASSESE, Sabino, «I beni culturali: dalla tutela alla valorizzazione», in Giornale di diritto amministrativo, 7/1998, pp. 673 et seq.; CASINI, Lorenzo, «Oltre la mitologia giuridica dei beni culturali», in Aedon. Rivista di arti e diritto online, 1-2/2012, URL:

< http://www.aedon.mulino.it/archivio/2012/1_2/casini.htm > [consultato il 1 agosto 2013].

3 RATTI, Emilio, ZATTERA Valeria, Storia e cronaca della bassa Val di Vara, Tivegna-Piana Battolla, Genova, Alkaest, 1988.

4 VENTURINI, Lorenzo (a cura di), Comunità, sacro e territorio in val di Vara: riflessioni e proposte interdisciplinari per la salvaguardia, la valorizzazione e l'uso delle risorse storiche, culturali e territoriali. Proposta di un sistema ecomuseale per la val di Vara: atti del convegno: Brugnato, 4 maggio 1996, La Spezia, Provincia della Spezia, Assessorato alla cultura, 1997.

5 RATTI, Marzia (a cura di), Arte e devozione in Val di Vara: catalogo della Mostra. Varese Ligure, Palazzo Cristiani-Picetti, 15 luglio-31 ottobre 1989, Genova, Sagep, 1989.
} 
Grazie a «[...] una multidirezionalità di contenuti collettivi corredati» ${ }^{6}$ si è riusciti a mettere in comunicazione università ed enti territoriali (Rocchetta di Vara, Beverino, Borghetto di Vara, Brugnato, Calice al Cornoviglio, Carro, Pignone, Riccò del Golfo e Zignago), coinvolgendo giovani ricercatori e studenti, conseguendo uno dei principali obbiettivi: «rendere disponibile in rete documenti, racconti e video del territorio attraverso una visione geografica supportata dalle nuove tecnologie e dai nuovi servizi di web mapping»7. Originariamente, infatti, il progetto prevedeva una ricognizione delle emergenze architettoniche $\mathrm{e}$ archeologiche che permettesse un'attenta ricognizione del territorio, finalizzata tanto all'analisi scientifica, quanto alla valorizzazione del patrimonio storico e culturale.

Il risultato è stato un complesso di ricerche interconnesse, che si è dipanato in cinque differenti ambiti.

La prima parte è stata dedicata allo studio del territorio, realizzando una mappa interattiva grazie all'utilizzazione di differenti softwares ${ }^{8}$, i cui risultati sono oggi visibili sul sito ufficiale del progetto 9 e in alcuni dei testi del volume.

La seconda parte del progetto è consacrata alla memoria orale. Il territorio della Val di Vara, è stato soggetto negli ultimi due secoli ad un forte processo di spopolamento: proprio per evitare che il patrimonio di usi e costumi che si tramandavano di generazione in generazione andasse perduto si è pensato di recuperare e conservare le memorie degli abitanti più anziani, depositari del ricordo di un modo diverso di vivere il territorio. Per far sì che il processo non si riducesse ad una mera operazione di immagazzinamento e conservazione di fonti finalizzate ad uno studio scientifico, ma potesse dar vita ad un processo riappropriativo, il procedimento è stato realizzato a partire da alcune premesse. In primo luogo la raccolta di dati è stata affidata a giovani studiosi, in modo da rendere gli anziani consapevoli del processo di trasmissione della memoria che veniva attuato; inoltre, le registrazioni delle memorie sono state fatte in un formato universalmente fruibile e riversate in un contenitore costantemente accessibile; infine l'opera di raccolta è sempre aperta e implementabile, possibilmente tramite apporti spontanei da parte degli stessi protagonisti: gli anziani ${ }^{10}$.

\footnotetext{
${ }^{6}$ SALVATORI, Enrica (a cura di), Storia e territorio della Val di Vara, Pisa, Felici, 2012, p. 147.

7 Ibidem, p. 186.

8 Il lavoro è stato diretto da Paolo Mogorovich dell'Istituto "A. Faedo" del CNR (Pisa) e realizzato da Claudio Schifani e Lorenzo Pini.

9 URL: < http://www.tramontivaldivara.it/il-progetto/ > [consultato il 5 giugno 2013].

10 Per compendiare queste tre fondamentali esigenze si è deciso di creare una squadra di raccolta delle testimonianze composta prevalentemente da giovani, diplomati e laureati di $18-25$ anni: «A questa squadra si è insegnato a fare una video intervista, a riversarne il contenuto su un computer, a procedere in un'opera di post-produzione di quanto registrato e infine a caricare i file prodotti sul portale con l'inserimento di opportuni metadati; parallelamente ai ragazzi è
} 
Nell'ambito del progetto sono stati recuperati anche alcuni lavori del documentarista Arturo Izzo: si tratta del documentario del 1990 Le pietre, $i$ muli, l'uomo: la viabilità medioevale in Val di Vara ${ }^{11}$, con testo di Isabella Ferrando e supervisione di Tiziano Mannoni, e di due ulteriori video relativi alla valle, uno del 1987 che consiste in un'intervista al corbaio Guido Bonati e il secondo, del 1988, in cui viene descritto il lavoro di Giovanni Guassone, l'ultimo mulattiere della Val di Vara.

La terza parte del progetto - che si esprime attraverso le pagine del volume Storia e territorio della Val di Vara - raccoglie i saggi storico-divulgativi e i percorsi sull'area oggetto di studio. Il primo tomo, di taglio scientifico, è affiancato da un secondo, che offre una serie di proposte di sentieri e percorsi nella Val di Vara, scelti da un lato per il loro valore paesaggistico, dall'altro per il fatto di toccare abitati e strutture interessanti dal punto di vista culturale.

I contributi di Storia e territorio sono dunque incentrati sul retaggio storico della valle. Il saggio Val di Vara: elementi per lo studio storico archeologico, dalla preistoria alla romanizzazione ${ }^{12}$ ricostruisce la storia delle ricerche archeologiche compiute nel corso degli anni nella Val di Vara e fornisce un compendio della preistoria e dei primi secoli di storia di questa porzione di territorio. Al testo si accompagnano alcuni approfondimenti - sviluppati all'interno di box, che costituiscono un vero e proprio apparato paratestuale - sulle località d'interesse archeologico. Medioevo in Val di Vara: problemi di racconto ${ }^{\mathbf{1 3}}$ non delinea una storia della Val di Vara nel medioevo, ma si sofferma sulla produzione storiografica locale e nazionale, su alcuni temi guida e sui molti problemi irrisolti, sui quali andrebbero approfondite le ricerche storiche. La valle, scrive Enrica Salvatori «è stata in passato - e in gran parte è ancora oggi - una sorta di ecosistema a sé, una zona peculiare e relativamente omogenea dal punto di vista naturalistico, geografico ed economico, pur in contatto con realtà insediative ed economiche differenti»14.

stata fornita e spiegata una scaletta ampia, ma coordinata, di domande [...]. Infine si è deciso di creare un portale web di tipo 2.0 che consentisse l'autocaricamento organizzato delle videointerviste, il loro commento e la loro fruizione tramite percorsi guidati ma personalizzati». Ibidem, p. 19.

${ }^{11}$ IZZO, Arturo, Le pietre, i muli, l'uomo: la viabilità medioevale in Val di Vara, Italia, 1990, 56 '.

${ }_{12}$ CAMPANA, Nadia, GERVASINI, Lucia, ROSSI, Stefano, Val di Vara: elementi per lo studio storico archeologico, dalla preistoria alla romanizzazione, in SALVATORI, Enrica (a cura di), Storia e territorio della Val di Vara, cit., pp. 33-107.

${ }_{13}$ SALVATORI, Enrica, Medioevo in Val di Vara: problemi di racconto, in SALVATORI, Enrica (a cura di), Storia e territorio della Val di Vara, cit., pp. 109-127.

14 Ibidem, p. 110. 
Massimo Grava ${ }^{15}$ analizza invece i destini dell'area in età moderna e contemporanea; la Val di Vara assunse un assetto amministrativo consolidato solo a partire dal 1923, quando nacque, con Regio Decreto di Vittorio Emanuele III, la provincia della Spezia. In questo caso risulta di particolare interesse l'incrocio delle fonti catastali e demografiche con quello dei sistemi dei GIS.

Per un'archeologia dei paesaggi in Val di Vara: documenti e materiali tra potenzialità e rischio di perdita cognitiva ${ }^{16}$ prende invece in esame lo sviluppo del paesaggio con il succedersi delle epoche, partendo dal presupposto che la storia sia produttrice di paesaggi (operando sui quadri ambientali naturali attraverso le azioni dell'uomo succedutesi nel tempo) e che la ricerca archeologica - sulla base di questa considerazione - debba essere impegnata a riconoscere, documentare, mettere in relazione tra loro e interpretare la costruzione del paesaggio. Per operare quest'analisi in rapporto all'età storica è necessario prevedere l'integrazione con le fonti, scritte ed orali.

Il saggio l'Atlante del patrimonio storico e culturale della Val di Vara17 illustra il funzionamento dell'Atlante inserito nel sito web del progetto "Tra Monti"; qui i sistemi territoriali vanno intesi come insieme di relazioni materiali ed immateriali.

L'Atlante è:

[...] uno strumento di ricerca e consultazione basato sulla rappresentazione delle identità storiche della Val di Vara attraverso una mappa interattiva. Può essere inteso come l'evoluzione di un catalogo o archivio di biblioteca in un nuovo catalogo in cui la ricerca non è più svolta scorrendo le lettere dell'alfabeto, ma osservando ed interrogando il territorio e le sue immagini. L'atlante non è statico poiché è lo stesso fruitore che ne costruisce di volta in volta nuove rappresentazioni attraverso le sue ricerche (es. cronologiche, tipologiche) ${ }^{18}$.

Il saggio successivo ${ }^{19}$ presenta più nel dettaglio proprio le caratteristiche del sito, che è da intendersi come una parte di un progetto di public history.

15 GRAVA, Massimiliano, La Val di Vara tra età moderna e contemporanea, SALVATORI, Enrica (a cura di), Storia e territorio della Val di Vara, cit., pp. 129-148.

16 BALDASSARRI, Monica, Per un'archeologia dei paesaggi in Val di Vara: documenti e materiali tra potenzialità e rischio di perdita cognitiva, in SALVATORI, Enrica (a cura di), Storia e territorio della Val di Vara, cit., pp. 149-184.

${ }_{17}$ MOGOROVICH, Paolo, PINI, Lorenzo, SCHIFANI, Claudio, L'Atlante del patrimonio storico e culturale della Val di Vara in SALVATORI, Enrica (a cura di), Storia e territorio della Val di Vara, cit., pp. 185-196.

18 Ibidem, pp. 190-191

19 BENEDETTI, Claudio, MANNARI, Chiara, TODARO, Elvira, Un sito web 2.0 per la Val di Vara, in SALVATORI, Enrica (a cura di), Storia e territorio della Val di Vara, cit., pp. 197-213. 
Punto di partenza indiscutibile è l'elemento territoriale, la Val di Vara, che attraverso il progetto si mira a promuovere, preservandone il patrimonio culturale e la memoria storica.

Lo scopo del sito è quello di fornire l'accesso al WebGIS con il patrimonio storicoarcheologico, consentire la visione delle interviste che costituiscono la memoria orale, rendere disponibile una bibliografia completa sulla valle e promuovere il territorio attraverso la pubblicazione di documentari e di itinerari trekking. [...] deve essere uno strumento a disposizione della comunità, il cui compito principale è quello di rafforzare le identità collettive, condividere memorie e promuovere il territorio ${ }^{20}$.

Il sito "Tra Monti" è suddiviso in quattro sezioni: Val di Vara che racconta il territorio attraverso testi, fotografie, video; Testimonianze che raccoglie la memoria orale della Val di Vara con le interviste rilasciate dagli abitanti anziani della valle; Biblioteca, che contiene un database di riferimenti bibliografici sulla Val di Vara; Alluvione, che contiene - sul modello dei più recenti archivi diffusi - fotografie, filmati e audio sull'alluvione che ha colpito la Val di Vara nel 2011.

Grazie al corredo di metainformazioni collegate a ciascun video, agli utenti è fornita la possibilità di creare percorsi personalizzati e di effettuare ricerche attive.

Per un'introduzione alle fonti archivistiche della Val di Vara di Riccardo Barotti ${ }^{21}$ è una breve rassegna delle fonti documentarie della valle; offre al lettore suggerimenti di ricerca, in particolare sull'età moderna e contemporanea.

Gli ultimi saggi ${ }^{22}$ di Storia e territorio, invece, si soffermano su specifici aspetti della storia della Val di Vara.

La quarta parte del progetto è rappresentata dal sito 2.0; come sottolinea Enrica Salvatori:

${ }^{20}$ Ibidem, p. 197.

21 BAROTTI, Riccardo, Per unintroduzione alle fonti archivistiche della Val di Vara, SALVATORI, Enrica (a cura di), Storia e territorio della Val di Vara, cit., pp. 215-224

${ }_{22}^{2}$ ROSSELLO, Marco, Ricerche storico-archeologiche nella media Val di Vara: il contributo della toponomastica, in SALVATORI, Enrica (a cura di), Storia e territorio della Val di Vara cit., pp. 225-230; GIORGIO, Marcella, Esperienze di ricognizione di superficie in Val di Vara. Difficoltà di lettura delle tracce archeologiche e perdita della memoria storica, in ibidem, pp. 231-236; MOSCATELLI, Damiano, Bozzolo: una roccaforte a difesa di Brugnato, in ibidem, pp. 237-246; DONATI, Piero, Sul patrimonio scultoreo della Val di Vara. L'arredo marmoreo delle chiese (secoli $X V-X V I$ ), in ibidem, pp. 247-268; GHELFI, Roberto, Su alcune dimore signorili della media Val di Vara, in ibidem, pp. 269-298; VALENZANO, Chiara, Sulle confraternite della Val di Vara, in ibidem, pp. 299-340. 
Una ventina di anni fa per documentare una ricerca di questo tipo si sarebbe scelto come unico veicolo di informazione il testo scritto: la relazione dettagliata e il volume scientifico. In sostanza il lavoro si sarebbe fermato alla parte appena descritta. Dieci anni fa probabilmente si sarebbe scelto di pubblicare almeno parte dei risultati anche sul web, in modo da dare maggiore pubblicità possibile a quanto fatto, mantenendo tuttavia come strumento principe di comunicazione e testimonianza la carta stampata. Oggi le cose stanno diversamente. Le tecniche, gli strumenti, i servizi e la filosofia stessa del web 2.0 consentono di pensare alla rete come principale e prioritario strumento di comunicazione non solo all'interno dell'équipe di studiosi, ma anche tra gli studiosi e le amministrazioni committenti, oltre che per la trasmissione delle conoscenze verso il grande pubblico e infine vera novità di questo nuovo medium - per la partecipazione stessa del pubblico al progetto 23 .

La rete, con i suoi strumenti, è stata la protagonista assoluta nel processo di attuazione del progetto ${ }^{24}$.

La mappa interattiva, oltre a svolgere il ruolo di piattaforma collaborativa per la ricerca, consente all'utente generico la costruzione di percorsi e indagini personalizzate e permette all'amministratore di avvalersi di uno strumento per la pianificazione degli interventi di facile aggiornamento e implementazione.

Il sito 2.o è finalizzato ad estendere le possibilità partecipative a tutti gli utenti, creando un elemento cementificante in grado di legare le comunità del territorio. Il progetto si ascrive agli invented archives, collezioni prive di un deposito fisico presso un ente deputato istituzionalmente alla raccolta e alla conservazione. L'organismo incaricato della conservazione della memoria condivisa diviene, i questi casi, un'entità evanescente, dai confini variabili: in questo caso, quelli della comunità locale.

La quinta parte, dedicata all'alluvione, è stata ideata in corso d'opera sulla base degli avvenimenti del 2011. Una sezione del sito, costruita in maniera non troppo dissimile da quella delle video-interviste, è stata consacrata a questo tema ed è stata avviata una campagna informativa per la raccolta del materiale.

L'operazione - già sperimentata in occasione di altri grandi eventi catastrofici rappresenta un caso unico - sinora - in Italia, in particolar modo per via della sua collocazione, all'interno di un piano più ampio, di progetto sorto per raccogliere il complesso delle memorie di un territorio.

${ }^{23}$ SALVATORI, Enrica (a cura di), Storia e territorio della Val di Vara, cit., p. 22.

${ }^{24} \mathrm{Si}$ veda il racconto dell'utilizzo di strumenti - come, ad esempio, Zotero o Dropbox - da parte della squadra impegnata nella realizzazione fattiva delle fasi di raccolta e immagazzinamento delle informazioni e delle fonti. 
Il complesso di materiali e strumenti di Tra Monti è da considerarsi infatti come un progetto di public history. espressione della volontà di interrogarsi sui bisogni di storia

che in maniera ricorrente emergono da singoli, gruppi, comunità; inoltre si deve anche cercare di rispondere a questi bisogni in maniera metodologicamente ineccepibile e contemporaneamente idonea alla richiesta, utilizzando a questo fine e con consapevolezza critica gli strumenti che ci arrivano dal mondo dell'ICT25.

Il progetto ambisce, dunque, senza perdere le caratteristiche di scientificità e non rinunciando alla capacità critica e all'applicazione di presupposti metodologici ineccepibili, a mantenere una natura contemporaneamente aperta e accessibile. La piattaforma per la costruzione e la condivisione di memorie, volta a conseguire il consolidamento e il recupero delle identità collettive è realizzata secondo i canoni propri della ricerca scientifica, pur conservando la possibilità di perseguire i presupposti espansivi ed estensivi del progetto (la raccolta di materiali è infatti sempre in corso ed è aperta ai contributi “esterni”).

25 SALVATORI, Enrica (a cura di), Storia e territorio della Val di Vara, cit., p. 23. 


\section{* L'autore}

Jacopo Bassi si è laureato in Storia della Chiesa presso l'Università di Bologna nel 2008 con una tesi sulla comunità ortodossa in Epiro e in Albania meridionale in età contemporanea.

URL: <http://www.studistorici.com/2009/02/24/jacopo_bassi >

\section{Per citare questo articolo:}

BASSI, Jacopo, «Recensione: Enrica SALVATORI, Storia e territorio della Val di Vara, Pisa, Felici, 2012, 344 pp.», Diacronie. Studi di Storia Contemporanea : Spazi, percorsi e memorie, 29/10/2013,

URL:<http://www.studistorici.com/2012/10/29/bassi_numero_15/ >

Diacronie Studi di Storia Contemporanea 0 www.diacronie.it

Risorsa digitale indipendente a carattere storiografico. Uscita trimestrale. redazione.diacronie@hotmail.it

Comitato di redazione: Marco Abram - Giampaolo Amodei - Jacopo Bassi - Luca Bufarale - Alessandro Cattunar - Alice De Rensis Barbara Galimberti - Deborah Paci - Fausto Pietrancosta - Matteo Tomasoni - Luca Zuccolo

Diritti: gli articoli di Diacronie. Studi di Storia Contemporanea sono pubblicati sotto licenza Creative Commons 2.5 Possono essere riprodotti a patto di non modificarne i contenuti e di non usarli per fini commerciali. La citazione di estratti è comunque sempre autorizzata, nei limiti previsti dalla legge. 